\section{Utilização de serviços de reabilitação pelas crianças e adolescentes dependentes de tecnologia de um hospital materno-infantil no Rio de Janeiro, Brasil}

\author{
Use of rehabilitation services by technology- \\ dependent children and adolescents in \\ a maternal and child hospital in \\ Rio de Janeiro, Brazil
}

\author{
Cláudia Zornoff Gavazza 1 \\ Vânia Matos Fonseca 1 \\ Kátia Silveira da Silva 1 \\ Sueli Rezende Cunha 1
}

\footnotetext{
1 Instituto Fernandes Figueira, Fundação Oswaldo Cruz, Rio de Janeiro, Brasil.

Correspondência C. Z. Gavazza Instituto Fernandes Figueira, Fundação Oswaldo Cruz. Av. Rui Barbosa 716, $4^{\circ}$ andar, Rio de Janeiro, $R J$ 22250-020, Brasil.

claudiagavazza@pop.com.br
}

\begin{abstract}
The objective of this study was to describe the dependence on technology and use of rehabilitation services by children and adolescents in a maternal and child hospital in Rio de Janeiro, Brazil. Using a cross-sectional design, the following variables were analyzed: gender and age of the children and adolescents, socioeconomic characteristics of the family, technology dependence, and use of rehabilitation services. The majority of the study population consisted of preschoolers (56.3\%), boys (58.3\%), residing in Greater Metropolitan Rio de Janeiro (89.3\%), from low-income families (70.9\%), and cared for mainly by their mothers (93.8\%), who in turn have low schooling (54.2\%) and are unemployed (89.6\%). Of the entire study population, $22.9 \%$ were dependent on more than three different technologies, with medication as the most prevalent. Government and nonprofit institutions fund the rehabilitation, and physical therapists are the most widely used health professionals during treatment (60.4\%). The target hospital provides all of the specialized medical treatment and most of the rehabilitation.
\end{abstract}

Rehabilitation Services; Biomedical Technology; Child; Adolescente

\section{Introdução}

Os adventos da revolução médico-tecnológica provocaram um aumento na incidência e na prevalência de doenças crônicas e no número de crianças dependentes de tecnologia para viver 1,2. Os dependentes de tecnologia são definidos como os que necessitam de um dispositivo tecnológico para substituir uma função vital do corpo, assim como cuidados continuados de enfermagem para afastar a morte ou futura incapacidade 3 . Essas crianças apresentam diversidades médico-clínicas que são refletidas pela incidência e gravidade das suas incapacidades associadas 4 e níveis de necessidade e complexidade de cuidados relativos ao dispositivo tecnológico que incorporam 5 .

Estudos realizados acerca dessa população específica, evidenciam uma falta de acompanhamento adequado de tais crianças após a alta hospitalar 6,7 sugerindo uma estrutura insuficiente da rede de assistência em suporte de reabilitação ${ }^{8,9}$.

Além disso, a descrição dos detalhes do acompanhamento reabilitacional das crianças está relacionada especialmente aos pacientes atendidos em regime de assistência domiciliar, normalmente dependentes de altas tecnologias como a ventilação mecânica 5,10. O perfil ambulatorial do acompanhamento reabilitacional encontrase associado a estudos realizados com crianças que apresentam atrasos do desenvolvimento ou 
que são portadoras de incapacidades 11,12, porém tal perfil não explicitava se as crianças possuíam algum tipo de dependência tecnológica ou não.

A falta de informações adequadas a respeito das características da dependência tecnológica, como da descrição das demandas por cuidados reabilitacionais dessa população específica, dificulta as ações de planejamento e organização dos serviços de assistência em saúde no que tange à integralidade dos cuidados.

Este estudo objetiva descrever as características da dependência tecnológica, como também as demandas reabilitacionais de crianças e adolescentes atendidas no campo ambulatorial, fora do regime domiciliar, cuja escolha está justamente na possibilidade de detectar o aparecimento de um perfil diferenciado de dependência e/ou demanda de cuidados, verificando, assim, até que ponto elas se assemelham independentemente da patologia, estado ou incapacidade da criança.

\section{Métodos}

Optamos por um estudo transversal, cujo campo de estudo foi realizado no hospital materno-infantil do Instituto Fernandes Figueira da Fundação Oswaldo Cruz (IFF/FIOCRUZ), que desenvolve atividades assistenciais de ensino e pesquisa. Essa unidade foi escolhida para o presente estudo, por desempenhar papel de centro de referência nas especialidades de neonatologia, cirurgia pediátrica e genética médica, recebe crianças com variados graus de gravidade e necessidade de cuidados.

Nossa amostra de estudo foi composta por 48 crianças e adolescentes entre 1 e 16 anos, em acompanhamento nos ambulatórios especializados do hospital, durante o período de maio a julho de 2006. O processo de seleção se deu por meio da escolha de ambulatórios com maior demanda de dependentes de tecnologia, e o tamanho da amostra foi estimado levando em conta a prevalência (63\%) da tecnologia mais utilizada pela população de estudo (derivação ventrículo-peritoneal), com nível de confiança de 99\% para um total de quarenta crianças. Escolhemos acrescentar $20 \%$ a mais de crianças com o intuito de proporcionar substituições para possíveis perdas. Os critérios de inclusão foram crianças e adolescentes dependentes de tecnologia na época do estudo, não internadas no hospital.

A coleta de dados foi realizada por meio de prontuários e aplicação de questionário respondido pelos pais das crianças nos dias em que compareciam ao hospital para a consulta ou atendimento ambulatorial. O questionário foi construído com itens de resposta abertos e fechados, subdividido em três partes. Na primeira parte, foram levantadas as características demográficas da criança (sexo, idade, regiões de origem), sócio-econômicas do cuidador (escolaridade, vínculo empregatício) e da família (renda familiar). Na segunda parte, foram coletadas a patologia de base e a dependência tecnológica (idade do início da dependência, número e tipo de dependências). E a especialidade do profissional de reabilitação, espaçamento entre as sessões de tratamento de reabilitação e serviço de reabilitação utilizado como última parte da pesquisa.

As variáveis sócio-econômicas (escolaridade e renda familiar) foram estratificadas segundo o Instituto Brasileiro de Geografia e Estatística (IBGE) e os tipos de dependência tecnológica definidas segundo critérios do Office of Technology Assessment dos Estados Unidos (OTA) ${ }^{3}$ e de Cunha ${ }^{13}$. A especialidade do profissional de reabilitação foi definida segundo as Portarias $n^{o}$. 303, 304 e 306 do Ministério de Saúde 14,15,16 que apontam os profissionais que constituem uma equipe de reabilitação. Os tipos de serviços de reabilitação foram estratificados em: hospital público (Sistema Único Saúde - SUS) [IFF/ FIOCRUZ]; filantrópico com convênio com o SUS [Associação Brasileira Beneficente de Reabilitação (ABBR), Associação Fluminense de Reabilitação (AFR) e Associação Pestalozzi]; filantrópico sem convênio com o SUS [Associação de Assistência à Criança Deficiente]; instituição particular; escola pública [municipal, estadual ou federal]; e outros, reconhecidos como: instituição pública não estatal sem convênio com o SUS [Rede Sarah de Hospitais de Reabilitação], e mais de uma das instituições citadas acima.

Para a construção e análise dos dados foi utilizado o programa Epi Info 2000 (Centers for Disease Control and Prevention, Atlanta, Estados Unidos).

O projeto deste estudo foi aprovado pelo Comitê de Ética do hospital em 5 de abril de 2006 sob o documento de registro $\mathrm{n}^{\circ}$. $059 / 05$. Os pais que optaram por participar da entrevista responderam ao questionário, assinaram um termo de consentimento livre e esclarecido após terem tomado conhecimento dos objetivos do estudo.

\section{Resultados}

\section{Características demográficas e} sócio-econômicas

A população de crianças e adolescentes dependentes de tecnologia é composta em sua maioria de pré-escolares $(56,3 \%)$ do sexo masculino (58,3\%), cuidados sobretudo pelas suas mães 
(93,8\%), que possuem escolaridade menor ou igual ao primeiro grau $(54,2 \%)$ e não trabalham (89,6\%). São oriundas de famílias com rendimentos mensais de até dois salários mínimos (70,9\%), residentes em sua maioria na região metropolitana do Estado do Rio de Janeiro (89,6\%), sendo $53 \%$ moradores de municípios diferentes do Município do Rio de Janeiro (Tabela 1).

\section{Características clínicas e dependência tecnológica}

A maioria da população estudada $(87,5 \%)$ é dependente de algum tipo de suporte medicamentoso. Pode-se observar que, de todas as dependências tecnológicas encontradas, houve uma predominância $(41,6 \%)$ daquelas relacionadas a algum grau de déficit, seqüela ou incapacidade relacionada ao sistema respiratório (Figura 1).
A dependência tecnológica iniciou-se numa fase bem precoce de vida, sendo metade dos indivíduos do nosso estudo já dependentes de algum tipo de tecnologia com menos de um mês de vida. Com relação à patologia de base, $70 \%$ estavam relacionadas a patologias congênitas, sendo $50 \%$ ligadas ao sistema nervoso central.

A complexidade dos cuidados dispensados a tal população também foi um marco a ser destacado, já que cerca de 56,3\% dos indivíduos apresentava de dois a quatro diferentes tipos de dependências tecnológicas.

\section{Utilização de serviços de reabilitação}

Toda a população de estudo realizava acompanhamento médico no hospital materno-infantil campo do estudo; 81,3\% já haviam recebido algum tipo de orientação por um profissional de

Tabela 1

Distribuição da população de estudo segundo características demográficas e sócio-econômicas. Instituto Fernandes Figueira, Fundação Oswaldo Cruz, Rio de Janeiro, Brasil, maio a julho de 2006.

\begin{tabular}{|c|c|c|}
\hline Variáveis & $n$ & $\%$ \\
\hline \multicolumn{3}{|l|}{ Faixa etária (anos) } \\
\hline $1-6$ & 27 & 56,3 \\
\hline $6-11$ & 15 & 31,3 \\
\hline $11-17$ & 6 & 12,5 \\
\hline \multicolumn{3}{|l|}{ Sexo } \\
\hline Masculino & 28 & 58,3 \\
\hline Feminino & 20 & 41,7 \\
\hline \multicolumn{3}{|l|}{ Cuidador principal } \\
\hline Mãe & 45 & 93,8 \\
\hline Avó & 2 & 4,2 \\
\hline Avô & 1 & 2,1 \\
\hline \multicolumn{3}{|l|}{ Escolaridade materna } \\
\hline Até 1o grau & 26 & 54,2 \\
\hline 2o grau & 22 & 45,8 \\
\hline \multicolumn{3}{|c|}{ Vínculo empregatício materno } \\
\hline Não trabalha & 43 & 89,6 \\
\hline Trabalha & 5 & 10,4 \\
\hline \multicolumn{3}{|c|}{ Renda familiar (salários mínimos) } \\
\hline Até 2 & 34 & 70,9 \\
\hline $2-10$ & 10 & 20,9 \\
\hline Sem declaração & 1 & 2,1 \\
\hline Sem rendimento & 3 & 6,3 \\
\hline \multicolumn{3}{|l|}{ Regiões de origem } \\
\hline Metropolitana & 43 & 89,6 \\
\hline Baixada litorânea & 3 & 6,3 \\
\hline Médio Paraíba & 1 & 2,1 \\
\hline Baía da Ilha Grande & 1 & 2,1 \\
\hline Total & 48 & 100,0 \\
\hline
\end{tabular}


Figura 1

Perfil das dependências tecnológicas encontradas na população de estudo. Instituto Fernandes Figueira, Fundação Oswaldo Cruz, Rio de Janeiro, Brasil, maio a julho de 2006 .

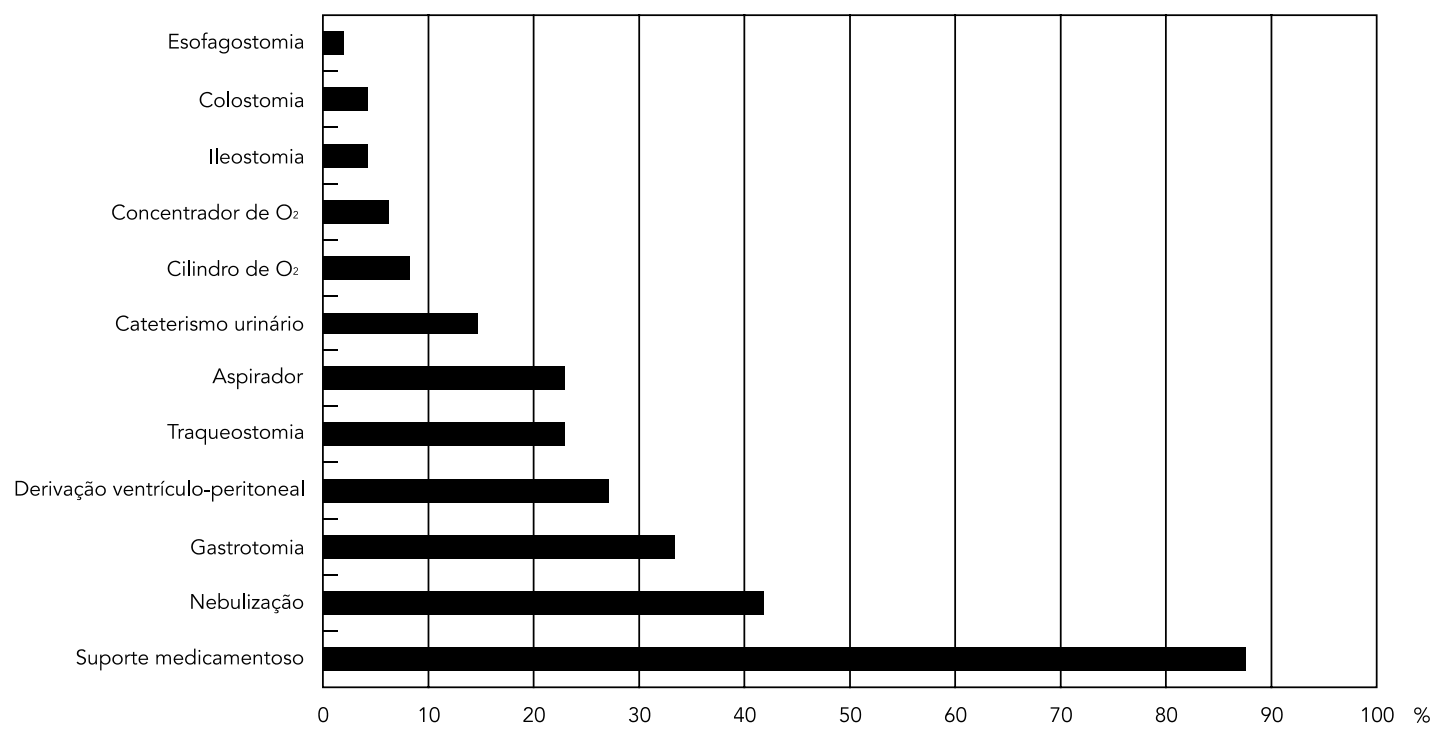

assistência social. Das 48 crianças estudadas, $81,3 \%$ foram identificadas em tratamento de reabilitação, ou seja, aquelas que estavam realizando acompanhamento de tratamento com, no mínimo, uma das seguintes especialidades terapêuticas: fisioterapia motora, fisioterapia respiratória, fonoaudiologia, terapia ocupacional, psicologia e enfermagem. Dessa maneira foi possível identificar que o fisioterapeuta motor $(60,4 \%)$ é o profissional mais utilizado no tratamento de reabilitação por essa população específica (Figura 2).

Os tratamentos de reabilitação (fisioterapia motora, fisioterapia respiratória, fonoaudiologia, psicologia, terapia ocupacional e enfermagem) são especialmente realizados em instituições filantrópicas conveniadas e não conveniadas ao SUS e no SUS (hospital público) (Tabela 2), por regimes de acompanhamento semanal ou bissemanal.

\section{Discussão}

A maioria das crianças (53\%) do nosso estudo reside em municípios diferentes do Município do Rio de Janeiro, onde se localiza o centro de referência para o tratamento dos cuidados específicos, obrigando o deslocamento da maior par- te das famílias para procurar um atendimento adequado. $\mathrm{O}$ fato coincide com os achados dos estudos qualitativos realizados com os familiares dos dependentes de tecnologia, que indicam que os pais referiam sentir-se sobrecarregados com os cuidados dedicados aos seus filhos, já que se viam obrigados a aprender a lidar sozinhos com a complexidade tecnológica e assistência necessária para a manutenção da vida de seus pequenos por causa da inexistência de profissionais especializados em tais cuidados próximos de seus domicílios 6,14,17,18. Além disso, os equipamentos específicos, necessários para os cuidados das crianças como ventiladores, aspiradores, cateteres, tubos de traqueostomia e medicamentos como antibióticos ou oxigenoterapia, não eram encontrados nos serviços comunitários, demonstrando a dificuldade de se encontrar redes de serviços de suporte adequados na comunidade local 9,19. Vale ressaltar que o presente trabalho foi realizado tendo como população de estudo a clientela de um hospital do SUS, referência na área maternoinfantil. O fato impõe limitações a este estudo, impedindo-nos de realizar validações externas, uma vez que nos proporciona uma visão restrita da realidade dessas crianças no Brasil, tanto pela inexistência de estudos nacionais a respeito, quanto pelo fato de este trabalho restringir-se a uma clientela de um hospital do SUS. 
Distribuição da população de estudo segundo a demanda por tipo de profissional de reabilitação. Instituto Fernandes Figueira, Fundação Oswaldo Cruz, Rio de Janeiro, Brasil, maio a julho de 2006.

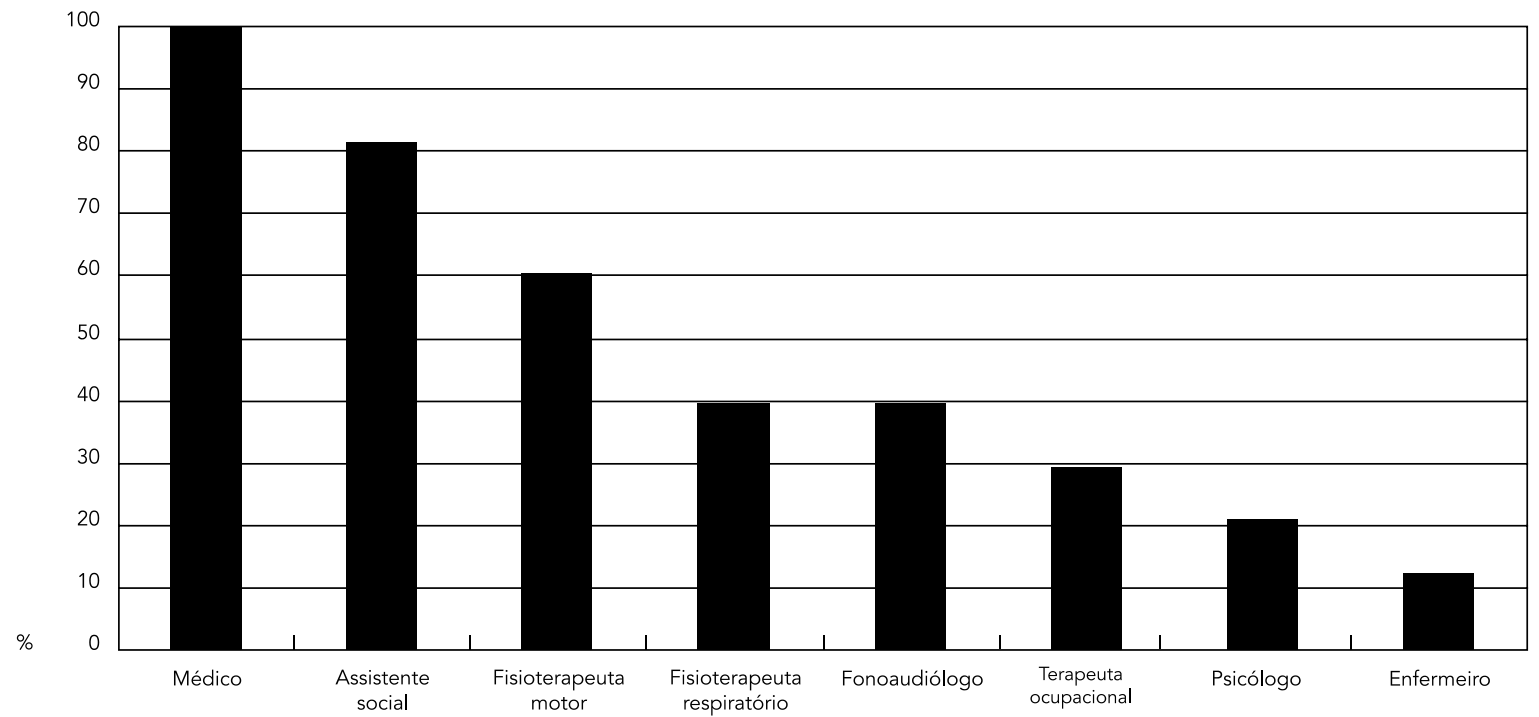

As variações regionais de serviços e profissionais especializados são conseqüências de desigualdades econômicas inter-regionais ${ }^{4}$ com reflexo sobre a capacidade local de financiamento, repercutindo assim no tamanho e complexidade da rede de serviços 20

Nossos achados evidenciam uma população de dependentes de tecnologia, acompanhados no campo ambulatorial, com doenças de base congênitas $(70 \%)$, relacionadas principalmente ao sistema nervoso central (50\%), cursando com desordens de desenvolvimento como as mielomielingoceles, encefalopatias, microcefalias e hidrocefalias. Os dados diferem dos estudos que descrevem a população de pacientes acompanhados em nível domiciliar porque esses são acometidos por doenças em maior parte ligadas ao sistema respiratório 5,10,21,22, dependentes de altas tecnologias como a ventilação mecânica. Vale comentar que este estudo optou por selecionar propositalmente a clientela de pacientes acompanhada em regime ambulatorial, que ocorreu durante todo o período selecionado para o campo, quando todas as crianças dependentes de tecnologia eram introduzidas no estudo. Assim sendo, a amostra foi realizada por conveniência e por isso não foram incluídas, provavelmente, as crianças que no momento do período selecionado para o campo deste estudo estariam internadas ou que por outros motivos vieram a faltar ou perder as consultas.

Com relação à dependência tecnológica, também foi observada uma diferença entre as nossas crianças, acompanhadas no campo ambulatorial, com relação às crianças que recebem cuidado domiciliar. A maioria de nossas crianças $(87,3 \%)$ depende de suporte medicamentoso; por conseguinte, podemos sugerir que elas apresentam necessidades menos intensas e especializadas de cuidados, podendo ser encaixadas na definição de Wagner et al. 23, como do grupo das "low techs".

Grande parte da população estudada (56,3\%) depende de dois a quatro diferentes tipos de tecnologias. Nossos achados coincidem com os dados já levantados em outros estudos, visto que a maior parte destas crianças depende de mais de um tipo de tecnologia para a sua sobrevivência 5,6,17,24.

No presente estudo, o início da dependência tecnológica ocorreu numa fase bem precoce (menos que um mês de vida) devido a doenças congênitas relacionadas particularmente ao sistema nervoso central. Dados levantados por outros estudos como o de Glendinning et al. 4, realizado em 2001 na Inglaterra, sugerem que o início da dependência tecnológica também deve ter ocorrido numa fase bem precoce de vida, entretanto por motivos clínicos diferentes, já que 
Distribuição da população de estudo segundo tipo de serviço de reabilitação. Instituto Fernandes Figueira, Fundação Oswaldo Cruz, Rio de Janeiro, Brasil, maio a julho de 2006

\begin{tabular}{|c|c|c|}
\hline Variáveis & $\mathrm{n}$ & $\%$ \\
\hline \multicolumn{3}{|l|}{ Serviço médico } \\
\hline Hospital público (SUS) & 48 & 100,0 \\
\hline \multicolumn{3}{|l|}{ Serviço de enfermagem } \\
\hline Hospital público (SUS) & 6 & 12,5 \\
\hline \multicolumn{3}{|l|}{ Serviço de assistência social } \\
\hline Hospital público (SUS) & 34 & 87,2 \\
\hline Filantrópico com convênio com o SUS & 3 & 7,7 \\
\hline Outros & 2 & 5,1 \\
\hline \multicolumn{3}{|l|}{ Serviço de fisioterapia motora } \\
\hline Filantrópico com convênio com o SUS & 9 & 31,0 \\
\hline Hospital público (SUS) & 6 & 20,7 \\
\hline Particular & 2 & 6,9 \\
\hline Filantrópico sem convênio com o SUS & 2 & 6,9 \\
\hline Escola pública & 1 & 3,4 \\
\hline Outros & 9 & 31,0 \\
\hline \multicolumn{3}{|l|}{ Serviço de fisioterapia respiratória } \\
\hline Hospital público (SUS) & 13 & 61,9 \\
\hline Filantrópico com convênio com o SUS & 3 & 14,3 \\
\hline Escola pública & 1 & 4,8 \\
\hline Outros & 4 & 19,1 \\
\hline \multicolumn{3}{|l|}{ Serviço de fonoaudiologia } \\
\hline Filantrópico com convênio com o SUS & 10 & 52,6 \\
\hline Hospital público (SUS) & 4 & 21,0 \\
\hline Filantrópico sem convênio com o SUS & 1 & 5,3 \\
\hline Escola pública & 1 & 5,3 \\
\hline Outros & 3 & 15,8 \\
\hline \multicolumn{3}{|l|}{ Serviço de terapia ocupacional } \\
\hline Hospital público (SUS) & 6 & 42,9 \\
\hline Filantrópico com convênio com o SUS & 2 & 14,3 \\
\hline Filantrópico sem convênio com o SUS & 2 & 14,3 \\
\hline Outros & 3 & 21,4 \\
\hline Escola pública & 1 & 7,1 \\
\hline \multicolumn{3}{|l|}{ Serviço de psicologia } \\
\hline Filantrópico com convênio com o SUS & 3 & 30,0 \\
\hline Filantrópico sem convênio com o SUS & 3 & 30,0 \\
\hline Outros & 4 & 40,0 \\
\hline
\end{tabular}

a maioria das crianças tinha dependências tecnológicas atreladas a conseqüências pulmonares em razão da prematuridade extrema.

Com relação às demandas por tratamentos de reabilitação, nossos achados evidenciam que o fisioterapeuta motor $(60,4 \%)$ é o profissional mais utilizado no processo. Tal achado diverge dos estudos realizados com os dependentes de tecnologia acompanhados em regime de "home care" 10 porque para estes o enfermeiro surge como maior demanda de tratamento em reabili- tação. No nosso trabalho apenas uma pequena parcela (12,5\%) encontra-se em assistência de enfermagem em base regular (uma vez ao mês ou duas vezes por semana). Esta diferença está relacionada à escolha do campo de estudo, que foi optado por ser composto de uma amostra de crianças em acompanhamento ambulatorial, justamente para levantarmos as diferenças e semelhanças em relação ao regime domiciliar.

Os serviços filantrópicos utilizados pela nossa população de estudo são tidos como serviços 
de referência para o atendimento ao portador de deficiência e saúde mental e estão concentrados na região metropolitana do Estado do Rio de Janeiro, na ABBR, na Associação Pestalozzi (Rio de Janeiro), na AFR, na Associação de Pais e Amigos dos Excepcionais (APAE) e na Associação Pestalozzi (Niterói). O fato parece indicar que as crianças residentes nessas regiões, por terem um melhor acesso geográfico aos serviços de referência em reabilitação, talvez estejam sendo mais bem assistidas em suas demandas reabilitacionais.

Todavia, não possuímos dados suficientes para esta suposição, uma vez que a organização de serviços de reabilitação, segundo Almeida 25, é fragmentada, verticalizada e centrada em patologias. Tal situação, segundo a autora, é justificada pela participação de segmentos ligados aos portadores de deficiência no SUS, que buscou atender com mais ênfase às demandas dessas camadas específicas que, organizadas, conseguiram impor seus desejos e direitos, fazendo assim com que os serviços assumissem uma área de concentração urbana e nas regiões economicamente ativas.

\section{Conclusões}

Nosso estudo mostrou que o hospital materno-infantil do IFF/FIOCRUZ concentrou todas as demandas médicas especializadas das crianças e adolescentes dependentes de tecnologia. Nesta constatação está a importância do IFF não somente como hospital de referência para as especialidades médicas, mas também como instituição difusora de encaminhamentos reabilitacionais. Para isto a instituição deve ter uma rede de suporte adequada de serviços para o tratamento reabilitacional. Isso reforça a necessidade de uma rede de cuidados regionalizada e hierarquizada de forma que os serviços estejam organizados em níveis crescentes de complexidade, circunscritos a uma determinada área geográfica e planejados mediante critérios epidemiológicos, com definição e conhecimento da clientela a ser atendida.

As características tecnológicas e as demandas por cuidados destinados a tal população devem ser criteriosamente analisadas por intermédio de mais levantamentos epidemiológicos, para a promoção de uma maior eqüidade na alocação de recursos e acesso da população às ações de saúde em todos os seus níveis de atenção.

\section{Resumo}

O objetivo deste estudo é caracterizar descritivamente a dependência tecnológica e a utilização de serviços de reabilitação pela população de crianças e adolescentes de um hospital materno-infantil do Rio de Janeiro, Brasil. O estudo transversal descreveu as características demográficas da criança e sócio-econômicas do cuidador e família, como também o tipo de dependência tecnológica e a utilização de serviços de reabilitação. A população do estudo é composta de pré-escolares (56,3\%), do sexo masculino (58,3\%), residentes na região metropolitana do Estado do Rio de Janeiro (89,6\%). São oriundos de famílias com rendimentos mensais até dois salários mínimos (70,9\%), cuidados sobretudo por suas mãe (93,8\%), que possuem escola- ridade menor ou igual ao ensino fundamental (54,2\%) e não trabalham (89,6\%). Dos entrevistados, um total de 22,9\% depende de três tipos diferentes de tecnologias, sendo o suporte medicamentoso $(87,5 \%)$ a mais utilizada. O tratamento de reabilitação é financiado preponderantemente pelo Sistema Único de Saúde (SUS) e instituições filantrópicas, sendo o fisioterapeuta motor $(60,4 \%)$ o profissional de maior demanda nesse tratamento. O hospital estudado concentra todos os atendimentos médicos especializados e a maior parte dos tratamentos em reabilitação.

Serviços de Reabilitação; Tecnologia Biomédica; Criança; Adolescente 


\section{Colaboradores}

C. Z. Gavazza participou de todas as fases do desenvolvimento da pesquisa: concepção, análise dos dados e elaboração da discussão dos resultados. V. M. Fonseca participou da montagem do banco de dados, análises estatísticas e revisão final do texto. K. S. Silveira participou do desenvolvimento da pesquisa, montagem do banco de dados e análises estatísticas. S. R. Cunha participou da revisão bibliográfica.

\section{Referências}

1. Wang KWK, Barnard A. Technology-dependent children and their families: a review. J Adv Nurs 2004; 45:36-46.

2. Stanley FJ. Health: centenary article - child health since federation. Canberra: Australian Bureau of Statistics; 2001. (Year Book Australia, 2001).

3. Office of Technology Assessment. Technology dependent children: hospital vs. home care - a technical memorandum Washington DC: U. S. Government Printing Office; 1987. (Report OTA-TM-H38).

4. Glendinning C, Kirk S, Guiffrida A, Lawton D. Technology-dependent children in the community: definitions, numbers and costs. Child Care Health Dev 2001; 27:321-34.

5. Mendes RAGCS. Programa de assistência domiciliar interdisciplinar do Instituto Fernandes Figueira: limites e possibilidades na construção de uma prática integral no cuidado à criança dependente de tecnologia [Dissertação de Mestrado]. Rio de Janeiro: Instituto Fernandes Figueira, Fundação Oswaldo Cruz; 2005.

6. Glendinning C, Kirk S. Developing services to supportparentscaringforatechnology-dependentchild at home. Child Care Health Dev 2004; 30:209-18.
7. Cunha SR. A enfermeira e a família da criança dependente de tecnologia: a intermediação dos saberes [Dissertação de Mestrado]. Rio de Janeiro: Escola de Enfermagem Anna Nery, Universidade Federal do Rio de Janeiro; 1997.

8. Meio MDBB, Magluta C, Mello RR, Moreira MEL. Análise situacional do atendimento ambulatorial prestado a recém-nascidos egressos das unidades de terapia intensiva neonatais do Rio de Janeiro. Ciênc Saúde Coletiva 2005; 10:299-307.

9. Leite NSL. A família da criança dependente de tecnologia: organização para a sobrevivência [Dissertação de Mestrado]. Rio de Janeiro: Instituto Fernandes Figueira, Fundação Oswaldo Cruz; 2003.

10. Aday LA, Wegener DH, Andersen RM, Aitken MJ. Home care for ventilator-assisted children. Health Aff (Millwood) 1989; 8:137-47.

11. Majnemer A, Shevell MI, Rosenbaum P, Abrahamowicz M. Early rehabilitation service utilization patterns in young children with developmental delays. Child Care Health Dev 2002; 28:29-37.

12. CanChild Center for Childhood Disability Research. Children with disabilities in Ontario: a profile of children's services. Hamilton: McMaster University; 2000. 
13. Cunha SR. A enfermeira-educadora, as Marias e o José: tecendo a rede de saberes e práticas sobre o cuidado à criança dependente de tecnologia na comunidade [Tese de Doutorado]. Rio de Janeiro: Escola de Enfermagem Anna Nery, Universidade Federal do Rio de Janeiro; 2001.

14. Ministério da Saúde. Portaria no . 303, de 2 de julho de 1992. Diário Oficial da União 1992; 3 jul.

15. Ministério da Saúde. Portaria no ${ }^{\circ} .304$, de 2 de julho de 1992. Diário Oficial da União 1992; 3 jul.

16. Ministério da Saúde. Portaria no. 306, de 2 de julho de 1992. Diário Oficial da União 1992; 3 jul.

17. Silva AC, Rodrigues KCS, Campos MA. Filhos da biotecnologia: traçando o perfil epidemiológico das crianças emergentes do Instituto Fernandes Figueira [Monografia de Especialização]. Rio de Janeiro: Instituto Fernandes Figueira, Fundação Oswaldo Cruz; 2003.

18. Kirk S. Caring for children with specialized health care needs in the community: the challenges for primary care. Health Soc Care Community 1999; 7:350-7.

19. Wheeler T, Lewis CC. Home care for medically fragile children: urban versus rural settings. Issues Compr Pediatr Nurs 1993; 16:13-30.
20. Travassos C. Equidade e o Sistema Único de Saúde: uma contribuição para debate. Cad Saúde Pública 1997; 13:325-30.

21. Carnevale FA, Alexander E, Davis M, Rennick J, Troini R. Daily living with distress and enrichment: the moral experience of families with ventilatorassisted children at home. Pediatrics 2006; 117: e48-60.

22. Noyes J, Hartmann H, Samuels M, Southall D. The experiences and views of parents who care for ventilator-dependent children. J Clin Nurs 1999; 8:440-50.

23. Wagner J, Power EJ, Fox H. Technology-dependent children: hospital versus home care. Philadelphia: J. P. Lippincott; 1988.

24. Heaton J, Noyes J, Sloper P, Shah R. Families' experiences of caring for technology-dependent children: a temporal perspective. Health Soc Care Community 2005; 13:441-50.

25. Almeida LGR. Estudo sobre a distribuição dos serviços de reabilitação: o caso do Rio de Janeiro [Dissertação de Mestrado]. Rio de Janeiro: Escola Nacional de Saúde Pública Sergio Arouca, Fundação Oswaldo Cruz; 2004.

Recebido em 10/Abr/2007

Versão final reapresentada em 18/Set/2007

Aprovado em 05/Out/2007 